\title{
Secondary prevention of stroke: Pleiotropic effects of optimal oral pharmacotherapy (Review)
}

\begin{abstract}
KIYOSHI KIKUCHI ${ }^{1,2,3}$, KO-ICHI KAWAHARA ${ }^{4,5^{*}}$, NAOKI MIURA ${ }^{6 *}$, TAKASHI ITO ${ }^{5}$, YOKO MORIMOTO ${ }^{7}$, SALUNYA TANCHAROEN $^{8}$, NOBUYUKI TAKESHIGE ${ }^{2}$, HISAAKI UCHIKADO $^{2}$, ROKUDAI SAKAMOTO $^{1}$, NAOHISA MIYAGI ${ }^{1}$, CHIEMI KIKUCHI ${ }^{9}$, NARUMI IIDA ${ }^{10}$, NAOTO SHIOMI ${ }^{11}$, TERUKAZU KURAMOTO ${ }^{12}$, MASARU HIROHATA ${ }^{2}$, IKURO MARUYAMA ${ }^{5}$, MOTOHIRO MORIOKA ${ }^{2}$ and EIICHIRO TANAKA ${ }^{3}$
\end{abstract}

${ }^{1}$ Department of Neurosurgery, Yame Public General Hospital, Yame 834-0034;

${ }^{2}$ Departments of Neurosurgery and ${ }^{3}$ Physiology, Kurume University School of Medicine, Kurume 830-0011;

${ }^{4}$ Laboratory of Functional Foods, Department of Biomedical Engineering, Osaka Institute of Technology, Osaka 535-8585; ${ }^{5}$ Department of Systems Biology in Thromboregulation, Kagoshima University Graduate School of Medical and Dental Sciences, Kagoshima 890-8520; ${ }^{6}$ Veterinary Teaching Hospital and Laboratory of Veterinary Diagnostic Imaging,

Faculty of Agriculture, Kagoshima University, Kagoshima 890-0065; ${ }^{7}$ Department of Restorative Dentistry and

Endodontology, Kagoshima University Graduate School of Medical and Dental Sciences, Kagoshima 890-8544, Japan;

${ }^{8}$ Department of Pharmacology, Faculty of Dentistry, Mahidol University, Rajthevee, Bangkok 10400, Thailand;

${ }^{9}$ Department of Rehabilitation, Nishida Koutoku Hospital, Saiki, Oita 876-0047; ${ }^{10}$ Research Laboratory, Kohjin Co., Ltd., Saiki, Oita 876-8580; ${ }^{11}$ Emergency Department, Saiseikai Shiga Hospital, Rittou, Shiga 520-3046;

${ }^{12}$ Department of Neurosurgery, Omuta City General Hospital, Omuta, Fukuoka 836-8567, Japan

Received October 14, 2011; Accepted January 6, 2012

DOI: $10.3892 / \mathrm{etm} .2012 .560$

\begin{abstract}
Stroke is a major cause of mortality and disability worldwide. During the past three decades, major advances have occurred in secondary prevention, which have demonstrated the broader potential for the prevention of stroke. Risk factors for stroke include previous stroke or transient ischemic attack, hypertension, high blood cholesterol and diabetes. Proven secondary prevention strategies are antiplatelet agents, antihypertensive drugs, statins and glycemic control. In the present review, we evaluated the secondary prevention of stroke in light of clinical studies and discuss new pleiotropic effects beyond the original effects and emerging clinical evidence, with a focus on the effect of optimal oral pharmacotherapy.
\end{abstract}

Correspondence to: Dr Kiyoshi Kikuchi, Department of Physiology, Kurume University School of Medicine, 67 Asahimachi, Kurume, Fukuoka 830-0011, Japan

E-mail:kkikuchi@m2.kufm.kagoshima-u.ac.jp

*Contributed equally

Key words: stroke, secondary prevention, oral pharmacotherapy, pleiotropic effects

\section{Contents}
1. Introduction
2. Antiplatelet agents
3. Antihypertensive drugs
4. Statins
5. Glycemic control
6. Conclusion

\section{Introduction}

Stroke is one of the leading causes of disability worldwide (1). In 2002, stroke-related disability was estimated to be the sixth most common cause of reduced disability-adjusted life-years (DALYs; the sum of life-years lost as a result of premature mortality and years lived with disability adjusted for severity) (2). However, due to the burgeoning elderly population in Western societies, it has been estimated that by 2030 , stroke-related disability in such societies will be the fourth most significant cause of DALYs (3). Stroke also has substantial costs related to complications, including poststroke dementia, depression, falls, fractures and epilepsy (4). Risk factors and sources of stroke must be identified in order to take steps towards preventing stroke (5). Although primary prevention is most significant in the reduction of stroke burden, effective secondary prevention is also essential (4). Secondary prevention addresses all measures for avoiding recurrences following a first transient ischemic attack (TIA) or 
stroke, which are becoming more frequent in an increasingly aging population (5). Risk factors for stroke include old age, hypertension, previous stroke or TIA, diabetes mellitus (DM), high blood cholesterol and tobacco smoking (6). Prevention of recurrent ischemic stroke may involve administration of antiplatelet agents, control and reduction of hypertension, and the use of 3-hydroxy-3-methylglutaryl-coenzyme A reductase inhibitors (statins) (6). Although similar oral agents exist in each category (antiplatelet agents, antihypertensives, statins and glycemic control), choosing the optimal agents from these categories could be significant for the secondary prevention of stroke.

\section{Antiplatelet agents}

The antiplatelet agents aspirin, clopidogrel and cilostazol have been demonstrated to be efficacious in the secondary prevention of stroke.

Aspirin prevents stroke among patients with recent stroke or TIA (7-9). Aspirin functions by irreversibly acetylating the cyclooxygenase enzyme; thus suppressing the production of thromboxane A2 and inhibiting the activation and aggregation of platelets (10). In a meta-regression analysis of placebo-controlled trials of aspirin therapy for secondary prevention of stroke, the relative risk reduction (RRR) for any type of stroke (hemorrhagic or ischemic) was estimated to be $15 \%$ [95\% confidence interval (CI), 6-23\%] (11). The magnitude of benefit is similar for doses 50-1,500 mg (7-9,12), although data for doses $<75 \mathrm{mg}$ are limited (13). The symptoms of toxicity also vary according to the administered dose. The principal toxicity of aspirin is gastrointestinal hemorrhage, and the greater the dose of aspirin, the greater the risk $(8,9)$. For patients using low-dose aspirin $(\leq 325 \mathrm{mg})$ for prolonged intervals, the annual risk of serious gastrointestinal hemorrhage is approximately $0.4 \%$, which is 2.5 -fold the risk for non-users $(8,9,14)$. Although aspirin therapy is associated with an increased risk of hemorrhagic stroke, the benefits of using low-dose aspirin for the prevention of ischemic stroke are greater. Therefore, there is a higher benefit-risk-ratio (15).

The antiplatelet agent, clopidogrel, effectively inhibits adenosine diphosphate-induced activation and aggregation of the platelets by selectively and irreversibly blocking the P2Y receptor (12) on the platelet membrane (14). Clopidogrel was studied in a randomized, blinded trial of clopidogrel vs. aspirin in patients at risk of ischemic events (CAPRIE) (14). In the study, 19,185 patients with ischemic stroke, myocardial infarction (MI) or peripheral vascular disease were randomized to $75 \mathrm{mg}$ of clopidogrel vs. $325 \mathrm{mg}$ of aspirin (14). Clopidogrel monotherapy proved to be more effective than aspirin monotherapy (RRR, 8.7; 95\% CI, 0.3-16.5; p=0.043) and is thus possibly more effective than aspirin against vascular disease affecting the heart and the peripheral circulation. However, the overall improvement in outcome was small and 108 patients needed to be treated in 2 years in order to prevent a major vascular event (stroke, MI or vascular death) (14).

Although the exact prevalence of antiplatelet resistance in ischemic stroke is not known, estimates concerning the two most widely used antiplatelet agents, aspirin and clopidogrel, suggest that it is high, irrespective of the definition used and the parameters measured (16). Inadequate antiplatelet respon- siveness correlates with an increased risk of recurrent ischemic vascular events in patients with stroke (16). Meanwhile, the addition of cilostazol has been demonstrated to reduce the biological resistance to aspirin and clopidogrel in clinical trials $(17,18)$.

In a previous study, Al-Qudah et al summarized the findings of major clinical trials and the efficacy of cilostazol in preventing ischemic stroke in comparison with other more commonly used antiplatelet medications, including aspirin and clopidogrel (19). Cilostazol is a relatively new antiplatelet agent that has been thoroughly investigated in Japan with promising outcomes for the secondary prevention of stroke $(12,19)$. The efficacy of cilostazol was revealed in certain major clinical trials (19). Cilostazol, a selective inhibitor of phosphodiesterase 3 (PDE3), prevents the inactivation of the intracellular second messenger cyclic adenosine monophosphate (cAMP), and irreversibly inhibits platelet aggregation and vasodilation (20). It has been used as a general antithrombotic agent and for stroke prevention in certain Asian countries (10). In the USA and certain European Union countries, cilostazol is only approved for the treatment of peripheral arterial disease (10).

Previously, the effectiveness of cilostazol $(100 \mathrm{mg}$, twice daily) compared with aspirin ( $81 \mathrm{mg}$, once daily) was examined in a randomized, double-blind pilot study that enrolled 720 patients with recent ischemic stroke (20). During 12-18 months of follow-up, stroke was observed in 12 patients assigned to cilostazol per year compared with 20 patients assigned to aspirin per year $(p=0.18)(20)$. Episodes of brain bleeding were significantly more common in the aspirin group than in the cilostazol group (7 vs. $1, \mathrm{p}=0.034)(20)$.

The aim of the cilostazol stroke prevention study (CSPS)2 (21) was to directly compare the efficacy and safety of cilostazol (100 mg, twice daily) and aspirin (81 mg, once daily) in secondary prevention of stroke in a total of 2757 Japanese patients. The incidence of the primary endpoint of stroke was low in the two groups, but was significantly lower in the cilostazoltreated group [hazard ratio (HR), 0.743; 95\% CI, 0.564-0.981; $\mathrm{p}=0.0357]$ (21). The incidence of hemorrhagic events was lower in the cilostazol group compared with the aspirin group (HR, 0.458; 95\% CI, 0.296-0.711; p=0.0004) (21). The cilostazolaspirin therapy against recurrent stroke with intracranial artery stenosis study (CATHARSIS) is an ongoing study. This study is evaluating the effect of aspirin (100 mg, daily) plus cilostazol (200 mg, daily), and aspirin (100 mg, daily) alone on the progression of intracranial arterial stenosis in 200 patients with chronic stroke and 50-99\% stenosis; the patients will be followed-up for up to 2 years (22). Thus, further data are required to assess the efficacy of cilostazol in stroke prevention in comparison with aspirin and clopidogrel (particularly in a multi-ethnic population) (10). According to various clinical studies of secondary prevention of stroke, cilostazol could be the most effective antiplatelet agent.

Several lines of evidence have demonstrated that cilostazol has pleiotropic neuroprotective effects on atherogenesis triggered by platelets. For example, atherogenesis is associated with the monocyte chemoattractant protein-1 (MCP-1), intercellular adhesion molecule (ICAM) and CD62P (P-selectin) (19). The central role of inflammation in the pathogenesis of atherothrombosis highlights the significance of inflammatory markers as prognostic tools (23). Several inflammatory markers, including 
(but not limited to) MCP1, ICAM and P-selectin, are associated with the progression of atherosclerotic lesions and thrombosis, as well as an increased risk of ischemic events (23). Based on experiments conducted on human umbilical vein endothelial cells, cilostazol was demonstrated to increase intracellular levels of cAMP and modulate the expression of MCP-1 in vascular endothelial cells (24). Cilostazol reduces the harmful actions of neutrophils by decreasing the expression of ICAM and P-selectin (25). Furthermore, cilostazol inhibits the interaction of platelets and leukocytes along with vascular endothelial cells, a role mediated by P-selectin (24). Additionally, cilostazol is neuroprotective through several mechanisms. Firstly, it increases the levels of $\mathrm{B}$-cell lymphoma $(\mathrm{Bcl})-2$, which is an antiapoptotic antioxidant promoter in the rat transient middle cerebral artery occlusion (MCAO) model (26). Secondly, it reduces the levels of the apoptotic protein, Bax, cytochrome $c$ and tumor necrosis factor (TNF)- $\alpha$, which mediate inflammatory and thrombotic effects in brain ischemia (26). Moreover, magnetic resonance imaging studies have revealed increased cerebral blood flow and cerebral blood volume following 2 and $24 \mathrm{~h}$ of MCAO in rats receiving cilostazol (27).

\section{Antihypertensive drugs}

As hypertension is a major risk factor for stroke (28), antihypertensive treatment is recommended for the secondary prevention of stroke. A systematic assessment of clinical data revealed that a significant reduction in recurrent stroke could be achieved with diuretics [odds ratio $(\mathrm{OR})=0.93 ; 95 \% \mathrm{CI}$, $0.5-0.92]$, and diuretics plus angiotensin-converting enzyme (ACE) inhibitors (OR=0.55; 95\% CI, 0.44-0.68), but not with $\beta$-blockers or ACE inhibitors alone (29). Although $\beta$-blockers are widely used as a first-line treatment for hypertension, a meta-analysis of 13 trials $(n=105,951)$ found a $16 \%(p=0.009)$ relative increase in the risk of stroke in patients using $\beta$-blockers as opposed to other antihypertensive agents (30).

For a number of patients, multiple antihypertensive therapies could confer great benefit (31). The tesults of the perindopril protection against recurrent stroke study (PROGRESS) have demonstrated that 6,105 patients with a prior stroke or TIA who received a combination of perindopril (ACE inhibitor) plus indapamide (thiazide diuretic) had a greater risk reduction of recurrent stroke compared with patients who received perindopril alone (43 vs. 5\%) (32).

Few clinical trials have directly compared various classes of blood pressure-lowering drugs following TIA or stroke (4). In the morbidity and mortality after stroke, eprosartan compared with nitrendipine for secondary prevention (MOSES) trial, 1,450 hypertensive patients with a stroke or TIA within the previous 2 years were randomly assigned to eprosartan [angiotensin II receptor blocker (ARB)] or nitrendipine (calcium-channel blocker) (33). Blood-pressure reductions were similar for the two agents, but the risk of stroke and TIA was lower with eprosartan [incidence density ratio (IDR), 0.75; 95\% CI, 0.58-0.97; p=0.03] (33). However, the majority of the benefit was due to fewer TIAs, with no significant effect on ischemic stroke (33).

The recently published ongoing telmisartan alone and in combination with ramipril global endpoint trial (ONTARGET) compared ramipril (ACE inhibitor), telmisartan (ARB) and a combination of the two drugs in 25,611 patients with vascular disease or high-risk DM over a median follow-up of 56 months (34). Approximately $21 \%$ of the patients in each treatment group had history of a stroke or TIA (34). Telmisartan, ramipril and the combination therapy proved to be equivalent with regard to the primary outcome parameter (mortality from cardiovascular causes, MI, stroke or hospitalization for heart failure) and prevalence of recurrent stroke (34). However, the prevention regimen for effectively avoiding second strokes (PRoFESS) trial failed to demonstrate a benefit for telmisartan in reducing the risk of recurrent stroke compared with a placebo (35). Therefore, evidence revealing the superiority of specific antihypertensive agents in the secondary prevention of stroke is currently insufficient.

\section{Statins}

In contrast to several large randomized studies demonstrating that statins significantly decrease recurrent coronary events in patients with coronary heart disease, only two trials using statins have been undertaken for the secondary prevention of TIA/stroke (36).

The heart protection study (HPS) compared simvastatin (40 mg/day) and a placebo in 20,536 patients with coronary artery disease, other occlusive vascular diseases (16\% of the study population had a history of TIA/stroke), DM, arterial hypertension or other risk factors, during a treatment period of 5 years (37). Although the trial demonstrated a significant overall reduction in stroke recurrence $(25 \%$; $95 \%$ CI, $0.15-0.34)$, the difference was not significant in the cohort of patients with pre-existing TIA/stroke (10.4 vs. 10.5\%) (37). However, patients with previous TIA/stroke demonstrated a significant reduction in the prevalence of any major vascular event (major coronary events, stroke and revascularization procedure) (37).

The stroke prevention by aggressive reduction in cholesterol levels (SPARCL) study compared atorvastatin and a placebo (38). The SPARCL trial was the first study dedicated to evaluating the role of statins in the secondary prevention of stroke (38). Overall, 4,731 patients who had experienced a TIA or minor stroke within the previous 6 months and had a level of low-density lipoprotein cholesterol of 100-190 mg/dl were randomized to receive a high dose of atorvastatin (80 mg/day) or a placebo. Following a mean period of 4.9 years, there was a $16 \%$ RRR in subsequent fatal or non-fatal stroke in patients treated with atorvastatin. The discrepancy in the prevalence of recurrent ischemic stroke between the HPS and SPARCL trials could be due to the fact that patients in the HPS were recruited (on average) 4.3 years following the initial vascular event, whereas it was only 6 months in the SPARCL trial (36).

Several lines of evidence have revealed that atorvastatin (a lipid-soluble HMG-CoA reductase inhibitor) has pleiotropic effects. Following short-term and low-dose therapy in patients with chronic cerebrovascular disease and hyperlipidemia, atorvastatin was revealed to lower lipid levels, significantly decrease collagen-induced platelet aggregation, significantly improve whole blood viscosity, deformability of red blood cells and activity of von Willebrand factor and improve hemorheological parameters, platelet aggregation and endothelial dysfunction (39). Furthermore, atorvastatin decreased markers 
of oxidative stress in hypercholesterolemic patients (40), and inhibited inflammatory angiogenesis in mice through the downregulation of vascular endothelial growth factor, TNF- $\alpha$ and transforming growth factor- $\beta 1$ (41). The complex beneficial effects of atorvastatin could prevent secondary stroke. According to various clinical studies of secondary prevention of stroke, atorvastatin could be the most effective statin.

\section{Glycemic control}

Individuals with DM have an increased risk of stroke (42). In the prospective pioglitazone clinical trial in macrovascular events (PROACTIVE) study (43), pioglitazone reduced fatal or non-fatal stroke (HR, 0.53; event prevalence, 5.6\% pioglitazone vs. $10.2 \%$ placebo), as well as other vascular events in a subgroup of 948 stroke patients within a total of 5,238 patients with type-2 diabetes mellitus (T2DM). Pioglitazone had pleiotropic effects in the patients with T2DM (44), including inhibiting oxidative stress, increasing adiponectin levels (45), and improving endothelial dysfunction in cerebral vessels (46). Beyond the hypoglycemic effects, the complex effects of pioglitazone could prevent secondary stroke. Since few trials have evaluated other glycemic control drugs following TIA or stroke, pioglitazone could be the most effective glycemic control agent.

\section{Conclusion}

As stroke is a frequent and severe disorder, and acute-stroke therapies (which are effective at the individual level) have only a limited impact on public health, secondary prevention of stroke is crucial. Vascular risk factors, including high blood pressure, high blood cholesterol and high blood glucose, should be treated to prevent ischemic stroke. To reduce the risk of new vascular events following a first ischemic stroke or TIA, a complementary strategy is the optimal management of the risk factors of stroke and TIA along with antithrombotic therapy. Secondary prevention with antiplatelet agents, antihypertensive drugs, statins and glycemic control as appropriate should be initiated urgently following TIA or stroke due to the high risk of early recurrence of stroke. Choosing the optimal oral pharmacotherapy in the secondary prevention of stroke is significant.

\section{Acknowledgements}

We wish to thank Dr Arshad Makhdum of the Edanz Group for editorial support.

\section{References}

1. Dobkin BH: Clinical practice. Rehabilitation after stroke. N Engl J Med 352: 1677-1684, 2005.

2. Murray CJ and Lopez AD: Global mortality, disability, and the contribution of risk factors: Global Burden of Disease Study. Lancet 349: 1436-1442, 1997.

3. Lopez AD, Mathers CD, Ezzati M, Jamison DT and Murray CJ: Global and regional burden of disease and risk factors, 2001: systematic analysis of population health data. Lancet 367: 1747-1757, 2006.

4. Rothwell PM, Algra A and Amarenco P: Medical treatment in acute and long-term secondary prevention after transient ischaemic attack and ischaemic stroke. Lancet 377: 1681-1692, 2011.
5. Paciaroni $\mathrm{M}$ and Bogousslavsky J: Primary and secondary prevention of ischemic stroke. Eur Neurol 63: 267-278, 2010.

6. Donnan GA, Fisher M, Macleod M and Davis SM: Stroke. Lancet 371: 1612-1623, 2008.

7. The Canadian Cooperative Study Group: A randomized trial of aspirin and sulfinpyrazone in threatened stroke. N Engl J Med 299: 53-59, 1978.

8. The Dutch TIA Trial Study Group: A comparison of two doses of aspirin (30 mg vs. $283 \mathrm{mg}$ a day) in patients after a transient ischemic attack or minor ischemic stroke. N Engl J Med 325: 1261-1266, 1991.

9. Farrell UK-TIA Study Group: The United Kingdom transient ischaemic attack (UK-TIA) aspirin trial: final results. J Neurol Neurosurg Psychiatry 54: 1044-1054, 1991.

10. Kral M, Herzig R, Sanak D, et al: Oral antiplatelet therapy in stroke prevention. Minireview. Biomed Pap Med Fac Univ Palacky Olomouc Czech Repub 154: 203-210, 2010.

11. Johnson ES, Lanes SF, Wentworth CE III, Satterfield MH, Abebe BL and Dicker LW: A metaregression analysis of the dose-response effect of aspirin on stroke. Arch Intern Med 159: 1248-1253, 1999.

12. Furie KL, Kasner SE, Adams RJ, et al: Guidelines for the prevention of stroke in patients with stroke or transient ischemic attack: a guideline for healthcare professionals from the american heart association/american stroke association. Stroke 42: 227-276, 2011.

13. Antithrombotic Trialists' Collaboration: Collaborative metaanalysis of randomised trials of antiplatelet therapy for prevention of death, myocardial infarction, and stroke in high risk patients. BMJ 324: 71-86, 2002.

14. CAPRIE Steering Committee: A randomised, blinded trial of clopidogrel versus aspirin in patients at risk of ischaemic events (CAPRIE). Lancet 348: 1329-1339, 1996.

15. He J, Whelton PK, Vu B and Klag MJ: Aspirin and risk of hemorrhagic stroke: a meta-analysis of randomized controlled trials. JAMA 280: 1930-1935, 1998

16. Topcuoglu MA, Arsava EM and Ay H: Antiplatelet resistance in stroke. Expert Rev Neurother 11: 251-263, 2011.

17. Lee JH, Cha JK, Lee SJ, Ha SW and Kwon SU: Addition of cilostazol reduces biological aspirin resistance in aspirin users with ischaemic stroke: a double-blind randomized clinical trial. Eur J Neurol 17: 434-442, 2011.

18. Maruyama H, Takeda H, Dembo T, et al: Clopidogrel resistance and the effect of combination cilostazol in patients with ischemic stroke or carotid artery stenting using the VerifyNow P2Y12 Assay. Intern Med 50: 695-698, 2011.

19. Al-Qudah ZA, Hassan AE and Qureshi AI: Cilostazol in patients with ischemic stroke. Expert Opin Pharmacother 12: 1305-1315, 2011.

20. Huang Y, Cheng Y, Wu J, et al: Cilostazol as an alternative to aspirin after ischaemic stroke: a randomised, double-blind, pilot study. Lancet Neurol 7: 494-499, 2008.

21. Shinohara Y, Katayama Y, Uchiyama S, et al: Cilostazol for prevention of secondary stroke (CSPS 2): an aspirin-controlled, double-blind, randomised non-inferiority trial. Lancet Neurol 9: 959-968, 2010.

22. Translational Research Informatics Center, Hyogo, Japan: Cilostazol-aspirin therapy against recurrent stroke with intracranial artery stenosis (CATHARSIS). 2009. http://www. strokecenter.org/trials/clinicalstudies/624. Accessed 10 January 2012.

23. Muhlestein JB: Effect of antiplatelet therapy on inflammatory markers in atherothrombotic patients. Thromb Haemost 103: 71-82, 2010.

24. Goto S: Cilostazol: potential mechanism of action for antithrombotic effects accompanied by a low rate of bleeding. Atheroscler Suppl 6: 3-11, 2005.

25. Omi H, Okayama N, Shimizu M, et al: Cilostazol inhibits high glucose-mediated endothelial-neutrophil adhesion by decreasing adhesion molecule expression via NO production. Microvasc Res 68: 119-125, 2004.

26. Hong KW, Lee JH, Kima KY, Park SY and Lee WS: Cilostazol: therapeutic potential against focal cerebral ischemic damage. Curr Pharm Des 12: 565-573, 2006.

27. Honda $\mathrm{F}$, Imai $\mathrm{H}$, Ishikawa $\mathrm{M}$, et al: Cilostazol attenuates gray and white matter damage in a rodent model of focal cerebral ischemia. Stroke 37: 223-228, 2006.

28. Lawes CM, Bennett DA, Feigin VL and Rodgers A: Blood pressure and stroke: an overview of published reviews. Stroke 35: 776-785, 2004. 
29. Rashid P, Leonardi-Bee J and Bath P: Blood pressure reduction and secondary prevention of stroke and other vascular events: a systematic review. Stroke 34: 2741-2748, 2003.

30. Lindholm LH, Carlberg B and Samuelsson O: Should beta blockers remain first choice in the treatment of primary hypertension? A meta-analysis. Lancet 366: 1545-1553, 2005.

31. Graham GD: Secondary stroke prevention: from guidelines to clinical practice. J Natl Med Assoc: 1125-1137, 2008

32. PROGRESS Collaborative Group: Randomised trial of a perindopril-based blood-pressure-lowering regimen among 6,105 individuals with previous stroke or transient ischaemic attack. Lancet 358: 1033-1041, 2001.

33. Schrader J, Luders S, Kulschewski A, et al: Morbidity and mortality after stroke, eprosartan compared with nitrendipine for secondary prevention: principal results of a prospective randomized controlled study (MOSES). Stroke 36: 1218-1226, 2005.

34. Yusuf S, Teo KK, Pogue J, et al: Telmisartan, ramipril, or both in patients at high risk for vascular events. N Engl J Med 358: 1547-1559, 2008.

35. Yusuf S, Diener HC, Sacco RL, et al: Telmisartan to prevent recurrent stroke and cardiovascular events. N Engl J Med 359: 1225-1237, 2008.

36. Weber R, Weimar C and Diener HC: Medical prevention of stroke and stroke recurrence in patients with TIA and minor stroke. Expert Opin Pharmacother 10: 1883-1894, 2009.

37. Collins R, Armitage J, Parish S, Sleight P and Peto R; Heart Protection Study Collaborative Group: Effects of cholesterollowering with simvastatin on stroke and other major vascular events in 20536 people with cerebrovascular disease or other high-risk conditions. Lancet 363: 757-767, 2004.

38. Amarenco P, Bogousslavsky J, Callahan A III, et al: High-dose atorvastatin after stroke or transient ischemic attack. N Engl J Med 355: 549-559, 2006

39. Szapary L, Horvath B, Marton Z, et al: Short-term effect of low-dose atorvastatin on haemorrheological parameters, platelet aggregation and endothelial function in patients with cerebrovascular disease and hyperlipidaemia. CNS Drugs 18: 165-172, 2004.
40. Ky B, Burke A, Tsimikas S, et al: The influence of pravastatin and atorvastatin on markers of oxidative stress in hypercholesterolemic humans. J Am Coll Cardiol 51: 1653-1662, 2008.

41. Araujo FA, Rocha MA, Mendes JB and Andrade SP Atorvastatin inhibits inflammatory angiogenesis in mice through down regulation of VEGF, TNF-alpha and TGF-beta1. Biomed Pharmacother 64: 29-34, 2010.

42. Selvin E, Coresh J, ShaharE, Zhang L, Steffes M and Sharrett AR Glycaemia (haemoglobin A1c) and incident ischaemic stroke: the Atherosclerosis Risk in Communities (ARIC) Study. Lancet Neur 4: 821-826, 2005.

43. Wilcox R, Bousser MG, Betteridge DJ, et al: Effects of pioglitazone in patients with type 2 diabetes with or without previous stroke: results from PROactive (PROspective pioglitAzone Clinical Trial In macroVascular Events 04). Stroke 38: 865-873, 2007.

44. Mori Y, Itoh Y, Obata T and Tajima N: Effects of pioglitazone vs. glibenclamide on postprandial increases in glucose and triglyceride levels and on oxidative stress in Japanese patients with type 2 diabetes. Endocrine 29: 143-148, 2006.

45. Fujitaka K, Otani H, Jo F, et al: Comparison of metabolic profile and adiponectin level with pioglitazone versus voglibose in patients with type-2 diabetes mellitus associated with metabolic syndrome. Endocrine 58: 425-432, 2011.

46. Petrica L, Vlad A, Petrica M, et al: Pioglitazone delays proximal tubule dysfunction and improves cerebral vessels endothelial dysfunction in normoalbuminuric patients with type 2 diabetes mellitus. Diabetes Res Clin Pract 94: 22-42, 2011. 